Dhaka Univ. J. Biol. Sci. 20(2): 183-189, 2011 (July)

\title{
GENOTYPE-SPECIFIC DETECTION OF GIARDIA LAMBLIA IN STOOL SAMPLES OF DIARRHOEAL AND NON-DIARRHOEAL PATIENTS IN DHAKA, BANGLADESH
}

\author{
Md. Masud Alam ${ }^{1}$, Mohammad Ilias*, Md. Abdullah Siddigue², Md. Mamun \\ $\mathrm{KABIR}^{2}$, FARIDA NAZIB ${ }^{2}$ AND MD. GULAM MuSAWWir KHAN ${ }^{2}$ \\ Department of Microbiology, University of Dhaka, Dhaka-1000, Bangladesh
}

Key words: Giardia lamblia, Genotypes, Multiplex real-time PCR, Immunoassay

\begin{abstract}
Two major genotypic assemblages (A and B) of Giardia lamblia infect humans. A single-vessel multiplex real-time PCR assay was used that genotypes Giardia infections into assemblages A and/or B directly from fecal samples. In this study, 157 diarrhoeal (symptomatic) and non-diarrhoeal (asymptomatic) stool samples collected from the International Centre for Diarrhoeal Disease Research, Bangladesh (ICDDR,B) and Bangabandhu Sheikh Mujib Medical University (BSMMU) hospital, respectively were analyzed to determine whether an association exists between infections with G. lamblia assemblages A or B and diarrhea in Bangladesh. Of the 157 stool samples, Giardia cysts were observed in 35 by microscopy and 127 showed positive result for Giardia cyst specific antigen. The 127 ELISA positive samples were assayed for genotyping by real-time polymerase chain reaction. Of the 117 real-time PCR positive stool samples, 15 were positive for G. lamblia assemblage A, 96 were positive for assemblage B and 6 samples showed positive result for both G. lamblia assemblage $A$ and $B$ infections. Higher ratios for diarrhea were observed for assemblage A infections, whereas higher parasite DNA loads and a higher overall rate were observed for assemblage $B$ infections in both diarrhoeal and non-diarrhoeal patients. Real-time PCR is, therefore, useful as an additional test supplementary to microscopy or enzyme immunoassay to detect genotypes of Giardia.
\end{abstract}

\section{Introduction}

The flagellated protozoan Giardia lamblia (synonymous with Giardia duodenalis, Giardia intestinalis) is an intestinal parasite that can infect many species in the animal kingdom including mammalian, avian and reptilian wildlife, domestic animals and humans.(1-2) In humans, G. lamblia can cause gastrointestinal infections ranging from mild to severe as well as chronic disease. Infection occurs by fecal oral route transmission, either by direct contact or by ingestion of contaminated food or water.(3) Despite morphological uniformity, considerable biotypic and genetic diversity exists within the G. lamblia species

\footnotetext{
*Corresponding author: <ilias14@yahoo.com>. 1Present address: Department of Microbiology, Noakhali Science and Technology University, Sonapur, Noakhali, Bangladesh. ${ }^{2}$ Parasitology Laboratory, International Centre for Diarrhoeal Disease Research, Bangladesh (ICDDR,B), Mohakhali, Dhaka, Bangladesh.
} 
The species includes several 'assemblages' or genotypes, A-G, that can be discriminated on the basis of host selection and genomic mutations.(4) Phylogenetic multilocus analysis using 18S rRNA, glutamate dehydrogenase (Gdh), elongation factor 1 alpha (EF-1 ) and triose phosphate isomerase (Tpi) gene based molecular methods have been used as representatives of each major genetic group to study the relations among assemblages from different hosts.(4-6) Phylogenetic sequence analysis of the independent genetic loci has provided essentially concordant results showing that there are two major G. lamblia group assmblages A and B which cause human infection. ${ }^{(7-8)}$

Routine diagnostic methods such as microscopy and enzyme-linked immunosorbent assay (ELISA) do not discriminate between the two assemblages of G. lamblia in human feces. Present authors therefore previously developed a multiplex real-time PCR assay utilizing self-probing amplicon primers that could distinguish assemblages A and B in a single reaction with sufficiently high sensitivity for high-throughput use directly on human fecal samples.(8) The present study was undertaken to genotype G. lamblia by multiplex real-time PCR assay and to compare multiplex real-time PCR with other (microscopy and ELISA) methods of diagnosis. They also observed the rate of G. lamblia assemblages A and B infection in symptomatic (diarrhoeal) and asymptomatic (nondiarrhoeal) patients in Bangladesh and the correlation between G. lamblia assemblage A and $B$ type infection in diarrhoeal and non-diarrhoeal patients.

\section{Materials and Methods}

Stool samples were collected for isolation of G. lamblia from both diarrhoeal and nondiarrhoeal patients. Case patients with diarrhoea admitted to the hospital of ICDDR,B in Dhaka were enrolled in this study as part of the routine surveillance of patients of all ages. Patients admitted to the BSMMU hospital, Dhaka, who did not have diarrhoea or had not taken antiprotozoal medication during the previous two months were enrolled as control patients in this study.

A total of 157 stool samples were collected in which 77 were diarrhoeal (case) and 80 were non-diarrhoeal (control) specimens. All fecal samples were tested to detect cysts (infectious) or trophozoite (non-infectious) of Giardia by saline wet-mount microscopy. Microscopy was conducted within a day of sample arrival at the initial diagnostic laboratory. Unpreserved fecal samples were first diluted twofold using normal saline (100 $\mu \mathrm{l}$ liquid stool specimen or $0.1 \mathrm{~g}$ of feces in $100 \mu \mathrm{l}$ normal saline). Diluted fecal samples were vigorously mixed by vortex mixer. One drop $(50 \mu \mathrm{l})$ of the diluted stool sample was placed on a slide and observed under a phase contrast microscope (Olympus, BX41TF, Japan) at different magnifications (40x and 100x) by saline wet mount method.

The enzyme linked immunosorbent assay (ELISA) was used for the qualitative detection of G. lamblia cyst antigen in human fecal samples by Giardia II ELISA kit 
(Techlab, Blacksburg) according to the manufacturer's instructions. Fecal samples were first diluted fivefold ( $100 \mu \mathrm{l}$ of liquid stool samples or $0.1 \mathrm{~g}$ of feces to $400 \mu \mathrm{l}$ of sample diluents) using sample diluent (supplied with the kit). The microassay plate in the kit contains immobilized monoclonal antibody specific for the cell surface antigen of $G$. lamblia. At first $100 \mu \mathrm{l}$ of sample diluent was transferred to each well except for positive and negative control wells. Then $50 \mu \mathrm{l}$ positive control, negative control (supplied with the kit) and diluted fecal samples were transferred to marked wells in the ELISA plate. The plate was sealed with a plate sealer and incubated for one hour at room temperature. The wells were washed thoroughly at least four times using the wash solution (supplied with the kit). After washing $50 \mu \mathrm{l}$ conjugate reagent (rabbit polyclonal antibody to a cell surface antigen of G. lamblia in a protein buffered solution containing $0.02 \%$ thimerosal) was added to each well and the plate was incubated $30 \mathrm{~min}$ at room temperature. The wells were washed by the same procedure after incubation with conjugate. Two drops $(100 \mu \mathrm{l})$ of substrate solutions (solution containing tetramethylbenzidine and peroxide) were added to each well and incubated at room temperature for 10 minutes. The reaction was stopped adding one drop $(50 \mu \mathrm{l})$ of stop solution in each well and the absorbance was measured at $450 \mathrm{~nm}$ using an ELISA plate reader. A sample was regarded as positive with an absorbance higher than 0.15 at $450 \mathrm{~nm}$ and negative when the absorbance was $\leq$ 0.15. Positive G. lamblia controls WB and GS were assemblages A-group 1 and assemblages B-group 3, respectively.

Fecal samples $(0.2 \mathrm{~g})$ were washed twice with sterile PBS and centrifuged (SIGMA D37520; Germany) for $5 \mathrm{~min}$ at 18,000x $\mathrm{g}$. The fecal pellet was subjected to six cycles of freeze-thaw in liquid nitrogen and at $95^{\circ} \mathrm{C}$ in a water bath. DNA was then extracted using a QIAamp DNA Stool Mini kit (QIAGEN, Valencia, Calif.) according to the manufacturer's instructions except that the suspension was incubated in the kit's stool lysis buffer at $95^{\circ} \mathrm{C}$ and a 3 min incubation with InhibitEx tablets was performed. The DNA was eluted in $0.2 \mathrm{ml}$ of AE buffer (supplied with the QIAGEN kit).

G. lamblia was detected by PCR using $18 \mathrm{~S}$ rRNA sequence specific primers and probes. Assemblage A-specific forward primer AF and assemblage B-specific forward primer BF were paired with the reverse primer R3 (common to both assemblage A and B sequences) (Table 1) to produce 95 and 102 bp products, respectively. Scorpion Uniprobes (Proligo, Paris, France) specific for assemblage A (ScA) and assemblage B (ScB) sequences were designed by Cherie et al.(8) (Table 1), whereby a 5 ' reporter dye hexachloro-6-carboxyfluorescein (HEX) for assemblage A and 6-carboxyfluorescine (FAM) for assemblage B, a specific stem-loop sequence, a black-hole quencher (BHQ1), and a hexethylene glycol (HEG) reverse-extension blocker were linked to the forward primers.

Multiplex PCR reactions were performed in $25 \mu \mathrm{l}$ with 1x PCR buffer (QIAGEN), 4.0 $\mathrm{mM} \mathrm{MgCl}$, $1.0 \mathrm{mM}$ of each deoxynucleoside triphosphates (dNTPs), $2.5 \mathrm{U}$ of Taq 
polymerase (HotStarTaq; QIAGEN), 6\% dimethyl sulfoxide (DMSO), $50 \mu \mathrm{g}$ of bovine serum albumin (BSA), 7.44 pmols of each Scorpion probe (ScA and ScB) and reverse primer (R3), and $5.4 \mu \mathrm{l}$ of DNA extracted from feces. Amplification was performed using an i-Cycler real-time detection system (Bio-Rad, Hercules, Calif) for $15 \mathrm{~min}$ at $95^{\circ} \mathrm{C}$ followed by 40 cycles of $30 \mathrm{sec}$ at $95^{\circ} \mathrm{C}, 30 \mathrm{sec}$ at $60^{\circ} \mathrm{C}$, and $30 \mathrm{sec}$ at $72^{\circ} \mathrm{C}$.

Table 1. Primers and probes used for detection of assemblage A and assemblage B of G. lamblia. Underlined sequences indicate the regions of stem loop sequences that are complementary to the neosynthesized strand.

\begin{tabular}{ll}
\hline $\begin{array}{l}\text { Name of primers/ } \\
\text { probes }\end{array}$ & Sequence $\left(5^{\prime} \mathbf{3}^{\prime}\right)$ \\
\hline AF & ATC CTG CCG GAG CGC GAC G \\
BF & CGG TCG ATC CTG CCG GAA TC \\
R3 & CGG GTG CAA CCG TTG TCC T \\
ScA & (HEX)CCCGGCGCATGGCTTCGTCCTTGCCGGG-BHQ1-HEG- \\
& ATCCTGCCGGAGCGCGACG \\
ScB & (FAM)CGGGCATGCATGGCCCG-BHQ1-HEG- \\
& CGGTCGATCCTGCCGGAATC \\
\hline
\end{tabular}

Values for the average cycle threshold $C_{T}$ (the number of PCR cycles required to detect a positive reaction) of real-time PCR amplification were compared between microscopy-positive and microscopy-negative groups by the Mann-Whitney test. The nonparametric correlation was used to quantitate the degree of association between ELISA optical density and $\mathrm{C}_{\mathrm{T}}$ value on ELISA-positive and real-time PCR-positive specimens.

\section{Results and Discussion}

Giardia trophozoites and/or cysts were observed in 35 of the 157 stool samples from diarrheal (11 of 77) and non-diarrhoeal (24 of 80) patients and the remaining 120 stool samples were microscopy negative. The rate of Giardia cyst detected by microscopy is $22 \%$.

Enzyme linked immunosorbent assay for G. lamblia trophozoite and/or cyst specific antigens showed positive result in 127 of the 157 stool samples from diarrhoeal (61 of 77) and non-diarrhoeal (66 of 80 ) patients. The rate of detection of G. lamblia by ELISA is about $81 \%$, suggesting it to be more sensitive than microscopy.

The 127 ELISA positive stool samples were analyzed by real-time PCR for detection of G. lamblia assemblage A and/or B infection. A total of 117 ELISA positive samples showed a positive result for G. lamblia either assemblage A or B or both A and B infection (Table 2). Of the 35 microscopy positive fecal samples, 3 (2 diarrhoeal and 1 nondiarrhoeal) were positive for assemblage A and 29 were (7 diarrhoeal and 22 non- 
diarrhoeal) positive for assemblage B infection (Table 2). Additionally, 2 microscopy positive diarrhoeal samples were positive for both assemblages A and B type G. lamblia infection.

Table 2. Detection of G. lamblia assemblage A and/ or B infection by real-time PCR in stool samples of diarrhoeal and non-diarrhoeal patients.

\begin{tabular}{|c|c|c|c|c|c|}
\hline \multirow[t]{2}{*}{ G. lamblia genotype } & \multicolumn{2}{|c|}{$\begin{array}{l}\text { Diarrhoeal Patients } \\
\qquad(\mathrm{n}=57)\end{array}$} & \multicolumn{2}{|c|}{$\begin{array}{l}\text { Non-diarrhoeal Patients } \\
\qquad(\mathrm{n}=60)\end{array}$} & \multirow[t]{2}{*}{ Total } \\
\hline & $\mathrm{M}^{+} \mathrm{E}^{+}$ & $M^{-} E^{+}$ & $\mathrm{M}^{+} \mathrm{E}^{+}$ & $M^{-} E^{+}$ & \\
\hline Assemblage A (quantity) & 2 & 12 & 1 & - & 15 \\
\hline Mean $C_{\text {т value }}$ & 37.1 & 35.96 & 31.6 & - & \\
\hline Median $C_{T}$ value & 37.1 & 37.85 & 31.6 & - & \\
\hline Assemblage B (quantity) & 7 & 32 & 22 & 35 & 96 \\
\hline Mean $C_{\text {T }}$ value & 32.9 & 35.60 & 31.82 & 34.93 & \\
\hline Median $C_{T}$ value & 33.4 & 36.95 & 32.80 & 35.90 & \\
\hline Mixed infection (quantity) & 2 & 2 & - & 2 & 6 \\
\hline \multicolumn{6}{|l|}{ Assemblage A } \\
\hline Mean $C_{\text {т value }}$ & 19.1 & 37.5 & - & 29 & \\
\hline Median $C_{T}$ value & 19.1 & 37.5 & - & 29 & \\
\hline \multicolumn{6}{|l|}{ Assemblage B } \\
\hline Mean $\mathrm{C}_{\mathrm{T}}$ value & 36.05 & 36.75 & - & 36.65 & \\
\hline Median $C_{T}$ value & 36.05 & 36.75 & - & 36.65 & \\
\hline & 11 & 46 & 23 & 37 & 117 \\
\hline
\end{tabular}

$\mathrm{M}^{+} \mathrm{E}^{+}=$Microscopic and ELISA positive. $\mathrm{M}^{-} \mathrm{E}^{+}=$Microscopic negative and ELISA positive

Of the 83 microscopy negative but ELISA positive (M-E') stool samples, 12 diarrhoeal samples were positive for G. lamblia assemblage A infection whereas 67 (32 diarrhoeal and 35 non-diarrhoeal) samples showed positive result for G. lamblia assemblage B infection (Table 2). The mean and median $C_{T}$ values for assemblage $A$ and $B$ in real-time $\mathrm{PCR}$ are shown in Table 2. Four $\mathrm{M}^{-\mathrm{E}^{+}}$stool samples (2 diarrhoeal and 2 non-diarrhoeal) were positive for both assemblages $\mathrm{A}$ and $\mathrm{B}$ infections.

The $C_{T}$ values of real-time PCR for diarrhoeal specimens exhibited negative correlation (correlation coefficient $=-0.325 ; \mathrm{p}=0.01$ by nonparametric correlation test) between G. lamblia assemblage A and B infections. There was a positive correlation between ELISA OD and $C_{T}$ value of G. lamblia assemblage B type in both diarrheal (correlation coefficient $=0.513 ; \mathrm{p}=0.01$ ) and non-diarrhoeal (correlation coefficient $=$ $0.321 ; p=0.01$ ) patients. Total correlation (correlation coefficient) in both diarrhoeal and non-diarrheal stool samples between ELISA and real-time PCR for assemblage A and B exhibited that ELISA OD value had a positive correlation with the assemblage B (correlation coefficient $=0.435 ; \mathrm{p}=0.01$ ) and a negative correlation with the assemblage A (correlation coefficient $=-0.027 ; \mathrm{p}=0.01$ ). The negative correlation (correlation coefficient 
$=-0.211 ; \mathrm{p}=0.01)$ of $\mathrm{C}_{\mathrm{T}}$ values between assemblages $\mathrm{A}$ and $\mathrm{B}$ indicates that most of the ELISA positive stool samples were caused by assemblage B in non-diarrhoeal infections.

The molecular characterization of Giardia spp. is useful for understanding the biology of the parasite, its host preferences, epidemiology and pathogenocity.(9) In this study, 34 of the 35 microscopy positive and 117 of the 127 ELISA positive stool samples showed positive results in real-time PCR for G. lamblia assemblage A and/or B infection. Ten ELISA positive fecal samples were negative by real-time PCR assay. This may be due to the lower specificity of ELISA method for cross reaction between G. lamblia assemblages A and B antigens with other Giardia strains. The high $C_{T}$ value $(>30)$ indicates weak positive results and should always be interpreted in relation to the clinical data. It is not clear whether the presence of small amount of DNA in patients stool correlate with disease, or indicate asymptomatic infection, which has been described previously for $G$. lamblia in a case control study in Netherlands. ${ }^{(10)}$ Further studies are needed to elucidate the relevance of the positive real-time PCR results with high $\mathrm{C}_{\mathrm{T}}$ values.

Assemblage identification by real-time PCR revealed that the prevalence of assemblage B infection was almost six times higher than that of assemblage A infection. There is a strong association of G. lamblia assemblage A with diarrhoeal and assemblage $B$ with non-diarrhoeal asymptomatic infections, which has been described previously for G. lamblia in a case control study in Bangladesh.(11,12) There is a negative correlation between assemblages A and B infections in both diarrhoeal and non-diarrhoeal patients. The high asymptomatic prevalence of assemblage B is consistent with the concept that only a minority of Giardia infection result in symptomatic disease.(13) It is assumed that the high endemicity of assemblage $B$ infection in patients provide some protection against assemblage $\mathrm{B}$ associated diarrhoea by producing immune response.

Microscopy remains the primary diagnostic method for detecting gastrointestinal parasites because of its ability to detect parasites other than G. lamblia.(14) However, ELISA is a valuable tool for the routine parasitological laboratory because of its speed and simplicity, especially when microscopic examinations of stools are not performed in the laboratory. When using the ELISA, samples still need to be processed by microscopy in order to detect other parasites and the small number of false negative G. lamblia stools. Multiplex real-time PCR assay has a higher sensitivity and specificity to detect specific type of microbe out of many organisms. This may eventually become the method of choice to detect the intestinal parasites.

\section{References}

1. Thompson RCA 2004. The zoonotic significance and molecular epidemiology of Giardia and giardiasis. Vet. Parasitol. 126: 15-35.

2. Appelbee AJ, RC Thompson and M Olson 2005. Giardia and Cryptosporidium in mammalian wildlife-current status and future needs. Trends Parasitol. 21: 370-376. 
3. Monis PT and RCA Thompson 2003. Cryptosporidium and Giardia-zoonosis: fact or fiction? Inf. Gen. Evol. 3: 233-244.

4. Monis PT, RH Andrews, G Mayrhofer and PL Ey 1999. Molecular systematics of the parasitic protozoan Giardia duodenalis. Mol. Biol. Evol. 16: 1135-1144.

5. van Keulen H, PT Macechko, S Wade, S Schaaf, PM Wallis and SL Erlandsen 2002. Presence of human Giardia in domestic, farm and wild animals, and environmental samples suggests a zoonotic potential for giardiasis. Vet. Parasitol. 108: 97-107.

6. Caccio SM, RCA Thompson, J McLaughlin and HV Smith 2005. Unraveling Cryptosporidium and Giardia epidemiology. Trends Parasitol. 21: 430-437.

7. Lu SQ, AC Baruch. and RD Adam. 1998. Molecular comparison of Giardia lamblia isolates. Int. J. Parasitol. 28:1341-1345.

8. Cherie TNg, CA Gilchrist, A Lane, S Roy, R Haque, and ER Houpt 2005. Multiplex real-time PCR assay using scorpion probes and DNA capture for genotype-specific detection of Giardia lamblia on fecal samples. J. Clin. Microbiol. 43(3): 1256-1260.

9. Adam RD 2001. Biology of Giardia lamblia. Clin. Microbiol. Rev. 14: 447-475.

10. De Wit MAS, MPG Koopmans, LM Kortbeek, NJ van Leeuwen, J Vinje and YTHP van Duynhoven 2001. Etiology of gastroenteritis in sentinel general practices in the Netherlands. Clin. Infect. Dis. 33: 280-288.

11. Haque R, S Roy, M Kabir, SE Stroup, D Mondol and ER Houpt. 2005. Giardia assemblage A infection and diarrhea in Bangladesh. J. Inf. Dis. 192: 2171-2173.

12. Haque R, D Mondal, A Karim, IH Molla, A Rahim, ASG Faruque, N Ahmad, BD Kirkpatrick, E Houpt, C Snider and WA Petri, Jr. 2009. Prospective case-control study of the association between common enteric protozoal parasites and diarrhea in Bangladesh. Clin. Infect. Dis. 48:1191-1197.

13. Ortega YR and RD Adam 1997. Giardia: overview and update. Clin. Infect. Dis. 25: 545-549.

14. Schuurman T, P Lankamp, A van Belkum, M Kooistra-Smid and A van Zwet 2007. Comparison of Microscopy, real-time PCR and a rapid immunoassay for the detection of Giardia lamblia in human stool specimens. Clin. Microbiol. Infect. 13: 1186-1191. 